\title{
Comportamiento frente al desgaste en materiales compuestos de aluminio reforzados con partículas cerámicas
}

\author{
D. BUSQUETS-MATAIX, N. MARTÍNEZ, V. AMIGÓ, M.D. SALVADOR, C. FERRER \\ Departamento de Ingeniería Mecánica y de Materiales. Universidad Politécnica de Valencia 46022-Valencia, España.
}

\begin{abstract}
El presente artículo trata sobre las propiedades tribológicas en materiales compuestos de matriz de aleación de aluminio AA6061, reforzados con partículas cerámicas de diferente naturaleza: $\mathrm{TiB}_{2}, \mathrm{~B}_{4} \mathrm{C}, \mathrm{Si}_{3} \mathrm{~N}_{4^{\prime}}, \mathrm{SiC}$ y $\mathrm{Al}_{2} \mathrm{O}_{3}$. Los materiales compuestos fueron obtenidos mediante un procedimiento de consolidación en estado sólido que combina la pulvimetalurgia con un proceso de extrusión en caliente.

El estudio tribológico realizado se basó en una metodología de ensayo del tipo pin-on-ring con deslizamiento en seco (sin lubricación). Se analizaron para cada caso el coeficiente de rozamiento y la tasa de desgaste en función de la distancia de deslizamiento. Asimismo, las muestras ensayadas se observaron mediante microscopía electrónica de barrido.

Los resultados obtenidos demuestran que las tasas de desgaste de los compuestos son inferiores en todos los casos a los de la matriz sin reforzar. Entre los compuestos, las diferencias encontradas son debidas al tamaño medio de las partículas de refuerzo, obteniéndose un menor desgaste para tamaños de refuerzo mayores, mientras que la naturaleza de los mismos no parece tener una marcada influencia. Con respecto al coeficiente de rozamiento, no se ha encontrado una tendencia parecida entre los compuestos estudiados, obteniéndose en unos casos un valor mayor y en otros menor que con respecto a la aleación sin reforzar.
\end{abstract}

Palabras clave: Compuestos de matriz de aluminio, refuerzos cerámicos, desgaste, tribología.

\section{Wear behaviour on ceramic particle reinforced aluminium matrix composites.}

The present work deals with the wear behaviour on AA6061 based aluminium matrix composites reinforced with different kinds of ceramic particles: $\mathrm{TiB}_{2}, \mathrm{~B}_{4} \mathrm{C}, \mathrm{Si}_{3} \mathrm{~N}_{4}, \mathrm{Al}_{2} \mathrm{O}_{3}$ y SiC. Composites were obtained by means of a solid-state consolidation method, combining both powder metallurgy processing and hot extrusion.

The study carried out was based on a pin-on-ring apparatus under dry sliding conditions (dry friction). Both friction coefficient and wear rate were measured for each composite. Scanning Electron Microscope (SEM) was used to analyse wear and the tribolayer formed.

Results show that wear rate is lower in composites than in the unreinforced alloy in every case, as expected. Differences among composites are probably related to different particle size rather than nature of reinforcements. On the other hand, friction coefficient do not show a trend on composites, being lower than the base alloy in some cases and higher in others.

Keywords: Aluminium matrix composites, ceramic reinforcement, wear, tribology.

\section{INTRODUCCIÓN}

En los últimos años se ha desatado un importante interés en las propiedades tribológicas de las aleaciones de aluminio debido a la potencial reducción de peso que se puede obtener en piezas y componentes de importancia en gran número de sectores industriales, sobretodo en la industria del automóvil. Aunque la resistencia al desgaste de las aleaciones de aluminio es pobre en general, pueden obtenerse una mejora sustancial con la introducción de refuerzos de elevada resistencia y dureza como pueden ser los de naturaleza cerámica. Es por ello que la utilización de materiales compuestos de matriz de aluminio (Aluminium Matrix Composites, AMCs) en aplicaciones que requieran un reducido peso y unas buenas características frente al desgaste puede tener un gran futuro.

El comportamiento tribológico a temperatura ambiente de los AMCs ha sido ampliamente estudiado para diferentes sistemas, tanto al considerar diferentes matrices (desde aluminio de pureza a diferentes aleaciones) como refuerzos: $\mathrm{Al}_{2} \mathrm{O}_{3}, \mathrm{SiC}, \mathrm{TiC}, \mathrm{TiB}_{2}, \mathrm{~B}_{4} \mathrm{C}$, etc $(1,2)$. Sin embargo, se debe de tener en cuenta que es difícil hacer una comparación cuantitativa entre los resultados obtenidos por los diferentes autores debido a que las tasas de desgaste y los coeficientes de fricción están muy influenciados por el sistema de ensayo (donde por ejemplo se utilizan los de "ball on disk", "pin on disc", "block on ring") y las variables del mismo (esfuerzo nominal de contacto, velocidad de des-

lizamiento, carga normal,...). Del mismo, modo, las diferentes formas de procesado de los compuestos, como la naturaleza de los mismos (aleación base, tipo, morfología y volumen de refuerzo) no proporcionan facilidades para la comparación directa de resultados (3).

Desde un punto de vista cualitativo se han obtenido una serie de conclusiones más o menos consensuadas. Así, la adición de relativamente pequeñas cantidades de partículas de $\mathrm{Al}_{2} \mathrm{O}_{3}$ (incluso menores que el 5\%) al aluminio y aleaciones Al-Si reducen significativamente la tasa de desgaste de estos materiales (4). Además, un cierto número de estudios han demostrado un aumento de la resistencia al desgaste en diversas matrices, tanto en condiciones de lubricación o en seco, tras la incorporación de refuerzo en forma de partículas o fibras de $\mathrm{SiC}$ $\mathrm{o} \mathrm{Al}_{2} \mathrm{O}_{3}$ (5-8). Así, Hosking et al. (5) mostraron que en el caso de una aleación de aluminio 2014 reforzada con un $20 \%$ de SiC con un tamaño de $16 \mu \mathrm{m}$ dio como resultado una reducción en el desgaste en seco contra acero (en un orden de 10) con relación a la matriz sin reforzar. Así mismo encontraron que el tamaño del refuerzo ejercía un efecto importante, en el sentido que tanto un mayor porcentaje como un mayor tamaño de refuerzo mejoraban las propiedades frente al desgaste. Aumentando la fracción volúmica de refuerzo hasta valores típicos entre 10 y $30 \%$ proporcionan una mejora cifrada en un factor entre 4 y $200(1,5,9,10)$. Ciertos estudios han corroborado estos resultados, 
concluyendo que dos de los factores más importantes que controlan la resistencia al desgaste son la fracción volúmica del refuerzo y su tamaño (5,10-12), aumentando con ambos.

Por otro lado, la relación entre la tasa de desgaste y el coeficiente de fricción no sigue una regla fija, por lo que no se puede establecer una correlación directa entre ellos. Aunque un valor de coeficiente de fricción bajo proporciona a menudo un desgaste menor, un incremento del primero no lleva aparejado un aumento del segundo en todos los casos. Al tener un coeficiente de fricción mayor, se incrementa así mismo la tasa de disipación de energía, pero esto puede hacer aumentar la temperatura del contacto (hasta un cierto punto) sin incrementar la tasa de desgaste, o puede resultar en un mayor desgaste del otro cuerpo en contacto (13).

Por lo tanto el presente estudio trata de establecer una comparación cuantitativa del comportamiento tribológico de diferentes AMCs reforzados tanto con partículas cerámicas tradicionales $\left(\mathrm{Al}_{2} \mathrm{O}_{3}\right.$ y $\left.\mathrm{SiC}\right)$ como con otras susceptibles de ser usadas como refuerzos $\left(\mathrm{B}_{4} \mathrm{C}, \mathrm{TiB}_{2}\right.$ y $\left.\mathrm{Si}_{3} \mathrm{~N}_{4}\right)$ y estudiadas previamente por los autores (14-16), sin las discrepancias en las distintas variables (procesado, aleaciones base, técnicas de ensayo,...) que aparecen al comparar los resultados de diversos autores.

\section{PROCEDIMIENTO EXPERIMENTAL}

\subsection{Materias primas}

La aleación de aluminio utilizada para la fabricación de los diferentes compuestos corresponde a la familia de aleaciones tratables térmicamente Al-Mg-Si, en concreto la AA6061 según la nomenclatura de la Aluminum Association americana. Su composición nominal aparece en la tabla I.

TABLA I. COMPOSICIÓN NOMINAL EN PORCENTAJE EN MASA DE LA ALEACIÓN DE ALUMINIO USADA COMO MATRIZ (AA6061).

\begin{tabular}{|c|c|c|c|c|c|}
\hline $\mathrm{Mg}$ & $\mathrm{Si}$ & $\mathrm{Fe}$ & $\mathrm{Cu}$ & $\mathrm{Cr}$ & $\mathrm{Al}$ \\
\hline 1.04 & 0.63 & 0.63 & 0.23 & 0.21 & Bal. \\
\hline
\end{tabular}

Los polvos de la AA6061 fueron suministrados por "The Aluminium Powder Co. Ltd." y fueron obtenidos mediante atomización por argón. Estos polvos tienen una morfología esférica característica, con un tamaño medio de partículas de $30 \mu \mathrm{m}$ e inferior a $100 \mu \mathrm{m}$.

Los refuerzos cerámicos utilizados para la fabricación de los distintos compuestos fueron los siguientes: $\mathrm{B}_{4} \mathrm{C}, \mathrm{TiB}_{2^{\prime}}, \mathrm{Si}_{3} \mathrm{~N}_{4^{\prime}} \mathrm{Al}_{2} \mathrm{O}_{3} \mathrm{y}$ $\mathrm{SiC}$. Los polvos de $\mathrm{TiB}_{2}$ y $\mathrm{Si}_{3} \mathrm{~N}_{4}$ fueron suministrados por "Advanced refractory technologies" mientras que los restantes lo fueron por "Alfa Aesar". Los distintos tamaños medios y morfología tipo de partícula vienen mostrados en la tabla II.

TABLA II. TAMAÑo DE PARTículA Y MORFOLOGía DE LOS REFUERZOS UTILIZADOS (P: POLIGONAL, E: TIPO ESCAMA)

\begin{tabular}{|c|c|c|c|c|c|}
\hline Refuerzo & $\mathrm{B}_{4} \mathrm{C}$ & $\mathrm{TiB}_{2}$ & $\mathrm{Si}_{3} \mathrm{~N}_{4}$ & $\mathrm{Al}_{2} \mathrm{O}_{3}$ & $\mathrm{SiC}$ \\
\hline Tamaño medio $(\mu \mathrm{m})$ & 17 & 9.4 & 6.6 & - & - \\
\hline Rango $(\mu \mathrm{m})$ & - & - & - & $0.9-2.2$ & 2 \\
\hline Morfología & $\mathbf{P}$ & $\mathbf{P}$ & $\mathbf{E}$ & $\mathbf{P}$ & $\mathbf{P}$ \\
\hline
\end{tabular}

\subsection{Proceso de fabricación}

Los compuestos fueron fabricados por un procedimiento combinado de pulvimetalurgia y extrusión en caliente, del cual aparece una descripción general en (17). Un resumen del mismo, con las variaciones adoptadas debido a la aleación y los refuerzos utilizados se describe a continuación. Los polvos de la aleación y cada uno de los refuerzos se mezclan en un molino de bolas durante 2 horas a 90 r.p.m. La mezcla se compacta uniaxialmente en frío hasta una presión de 250 Mpa para obtener una pieza cilíndrica en verde con $25 \mathrm{~mm}$ de diámetro. A continuación, se procede al calentamiento del mismo hasta una temperatura de $803 \mathrm{~K}$ y se extruye con una relación de 25:1. De esta forma se obtienen barras cilíndricas de $5 \mathrm{~mm}$ de diámetro y de longitud variable (200 400 mm). Este procedimiento de fabricación de compuestos de matriz de aluminio se ha mostrado como muy eficaz, obteniendo una buena distribución de los refuerzos por toda la matriz y consolidación del material en la mayoría de los casos.

Se extruyeron compuestos con distintos porcentajes de refuerzo en volumen $(5,10$ y $15 \%$ ) aunque el presente estudio se centra en los compuestos de mayor fracción volúmica (15\%), por las razones expuestas en la introducción.

Asimismo, los materiales se trataron térmicamente para obtener el estado T6 de máximo endurecimiento. Este tratamiento consistió en la solubilización a $530^{\circ} \mathrm{C}(803 \mathrm{~K})$ durante 1 hora, temple en agua y posterior envejecimiento a $175^{\circ} \mathrm{C}(448 \mathrm{~K})$ durante 8 horas.

\subsection{Técnicas de ensayo y análisis.}

Los ensayos de desgaste fueron realizados en un aparato experimental del tipo "pin-on-ring" en donde una muestra del material a ensayar se hace deslizar en seco contra un anillo de acero cromado. La muestra cilíndrica, de $11 \mathrm{~mm}$ de longitud, apoya en una de sus bases en la superficie externa del anillo. Mediante un par de células de carga y un sistema de adquisición de datos computerizado se puede medir el coeficiente de rozamiento en cada momento. El desgaste del material viene evaluado cada cierto tiempo mediante diferencia de peso. Los ensayos fueron realizados bajo una carga constante de $136 \mathrm{~N}$ y una velocidad de deslizamiento de $1 \mathrm{~m} / \mathrm{s}$. La distancia total recorrida para cada uno de los casos fue de $2000 \mathrm{~m}$.

Estos estudios fueron completados mediante microscopía electrónica de barrido en donde se analizaron las huellas y deformaciones producidas en las superficies de contacto de los compuestos después de los ensayos. Las muestras fueron cortadas longitudinalmente a través de un plano perpendicular al movimiento relativo muestra/anillo y preparadas metalográficamente para tal efecto.

\section{RESULTADOS.}

Se realizaron ensayos de tracción en las muestras de los distintos materiales compuestos y de la matriz base en estado T6. Los resultados de resistencia máxima a la tracción y alargamiento proporcional de rotura aparecen en la tabla III.

TABLA III. PROPIEDADES MECÁNICAS DE LOS MATERIALES ESTUDIADOS.

\begin{tabular}{|c|c|c|}
\hline Material & $\sigma_{\mathrm{u}}(\mathbf{M P a})$ & $\varepsilon(\%)$ \\
\hline $\mathrm{AA6061} \mathrm{T} 6$ & 322 & 12 \\
\hline$+\mathrm{B}_{4} \mathrm{C}$ & 309 & 7 \\
\hline$+\mathrm{TiB}_{2}$ & 317 & --- \\
\hline$+\mathrm{Si}_{3} \mathrm{~N}_{4}$ & 369 & 11 \\
\hline$+\mathrm{Al}_{2} \mathrm{O}_{3}$ & 276 & 13 \\
\hline$+\mathrm{SiC}$ & 357 & 10 \\
\hline
\end{tabular}

Los valores obtenidos para la matriz sin reforzar se corresponden con los valores de la literatura (18) para esta aleación en el estado T6. Sin embargo en el caso de los materiales compuestos, se observa un comportamiento dispar según el refuerzo considerado. En el caso del refuerzo con partículas de $\mathrm{B}_{4} \mathrm{C}$ y $\mathrm{TiB}_{2}$ la resistencia mecánica se 
ve ligeramente disminuida con respecto a la aleación base, aunque se puede considerar que sea del mismo orden. Sin embargo, y como era de esperar, se encuentran unos valores de alargamiento inferiores con respecto a la matriz, debidos a la disminución de la ductilidad que conlleva el refuerzo partículas en este tipo de materiales. Por otro lado, para los materiales compuestos reforzados con $\mathrm{Si}_{3} \mathrm{~N}_{4}$ y SiC se obtienen unos incrementos importantes en la resistencia máxima con respecto a la matriz, y lo que es más importante, sin una merma apreciable en los valores de alargamiento. En ambos casos este valor se sitúa por encima del $10 \%$, lo que es indicativo de una muy buena ductilidad para este tipo de materiales. Por último cabe destacar el caso del material compuesto reforzado con $\mathrm{Al}_{2} \mathrm{O}_{3^{\prime}}$ en el que se observa una disminución importante de la carga de rotura con respecto a la aleación base.

A continuación se muestran los resultados de los ensayos de desgaste realizados. En la figura 1 se representa la pérdida de peso (ordenadas) con respecto a la distancia de deslizamiento seco recorrida (abscisas). Se puede apreciar como la pérdida de peso en todos los casos sigue una tendencia lineal creciente, por lo que es posible calcular la tasa de desgaste con bastante precisión. Estos valores calculados vienen tabulados en la tabla IV. En ésta se puede apreciar cómo en todos los materiales compuestos tienen una tasa de desgaste inferior que el material base. Sin embargo, entre ellos hay diferencias importantes. En efecto, para los materiales compuestos reforzados con partículas del tipo $\mathrm{Al}_{2} \mathrm{O}_{3}$ y $\mathrm{SiC}$ se obtienen tasas de desgaste alrededor de un $30 \%$ inferiores al material base. Sin embargo, en el caso de los materiales compuestos reforzados con $\mathrm{B}_{4} \mathrm{C} \mathrm{y} \mathrm{Si}_{3} \mathrm{~N}_{4}$ esta disminución alcanza un $60 \%$ aproximadamente. Hay que puntualizar que al tasa de desgaste obtenida para el compuesto reforzado con partículas de $\mathrm{TiB}_{2}$ corresponde a un porcentaje de refuerzo inferior $(10 \%)$ que en los otros casos, debido a problemas experimentales en la realización de las experiencias con un contenido de refuerzo superior (15\%).

El estudio tribológico de los compuestos se completó con la medición del coeficiente de rozamiento instantáneo durante toda la duración del ensayo de desgaste, mediante el procedimiento indicado en la parte experimental. Una gráfica tipo de los datos adquiridos mediante el equipo se muestra en la figura 2. En esta se puede observar el registro del coeficiente de rozamiento en cada momento del ensayo en función de la distancia recorrida. Para cada uno de los materiales estudiados, y a partir de los registros del coeficiente de rozamiento se hallaron la media y la desviación típica de todas las medidas (tabla V). Es de destacar que no se aprecian cambios bruscos en la media debidos a cambios sustanciales en el mecanismo de desgaste salvo por una pequeña etapa al inicio debida a las condiciones transitorias iniciales de formación de la capa de deformación tribológica (tribolayer). Estos cambios bruscos podrían aparecer como consecuencia de un incremento importante de la temperatura después de un determinado momento del ensayo, incrementando en gran medida el degaste y afectando al coeficiente de rozamiento al aumentar las fuerzas de cohesión. Por lo tanto, dados los resultados obtenidos, el estudio estadístico realizado resulta adecuado.

TABLA IV. TASAS DE DEsGaste.

\begin{tabular}{|c|c|}
\hline Material & Desgaste (g/m) $\mathbf{x} \mathbf{1 0}^{\mathbf{5}}$ \\
\hline $\mathrm{AA} 6061 \mathrm{~T} 6$ & 1.60 \\
\hline$+\mathrm{B}_{4} \mathrm{C}$ & 0.45 \\
\hline$+\mathrm{TiB}_{2}$ & 1.13 \\
\hline$+\mathrm{Si}_{3} \mathrm{~N}_{4}$ & 0.47 \\
\hline$+\mathrm{Al}_{2} \mathrm{O}_{3}$ & 0.96 \\
\hline$+\mathrm{SiC}$ & 1.03 \\
\hline
\end{tabular}

TABLA V. COEFICIENTE DE ROZAMIENTO EN SECO.

\begin{tabular}{|c|c|c|}
\hline Material & $\mu$ & Desv. est. \\
\hline $\mathrm{AA6061} \mathrm{T} 6$ & 0.327 & 0.031 \\
\hline$+\mathrm{B}_{4} \mathrm{C}$ & 0.318 & 0.096 \\
\hline$+\mathrm{TiB}_{2}$ & 0.322 & 0.117 \\
\hline$+\mathrm{Si}_{3} \mathrm{~N}_{4}$ & 0.245 & 0.106 \\
\hline$+\mathrm{Al}_{2} \mathrm{O}_{3}$ & 0.437 & 0.066 \\
\hline$+\mathrm{SiC}$ & 0.385 & 0.037 \\
\hline
\end{tabular}

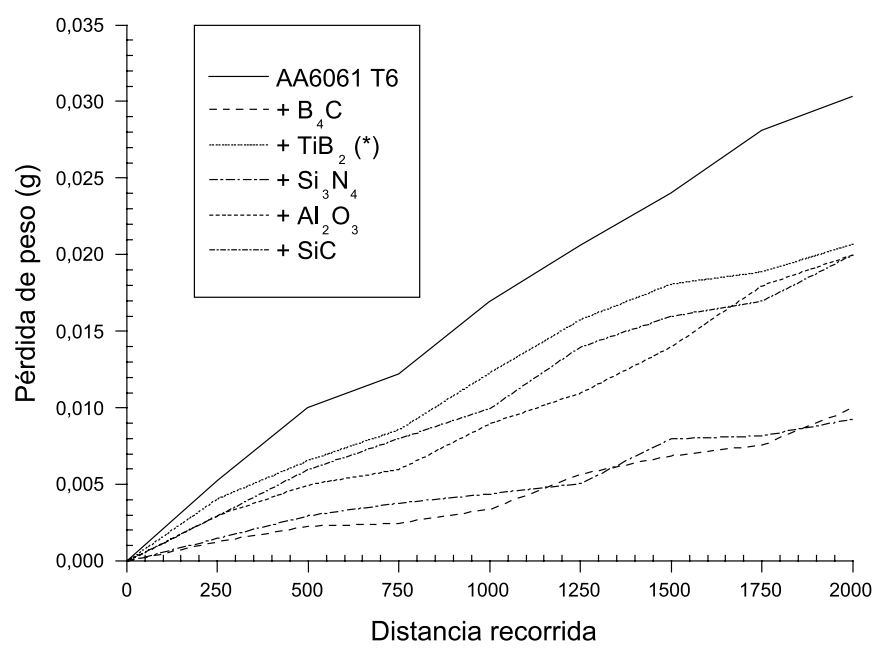

Figura 1. Pérdida de peso acumulada en función de la distancia de deslizamiento para todos los materiales estudiados.



Figura 2. Evolución del coeficiente de rozamiento con respecto a la distancia recorrida para el compuesto reforzado con $\mathrm{SiC}$.

Con respecto a los mecanismos de desgaste, todas la muestras se estudiaron mediante microscopía electrónica de barrido (SEM) y análisis de rayos $\mathrm{X}$ por energías dispersivas (EDX). La figura 3 se muestra una sección transversal a la dirección de deslizamiento del material compuesto reforzado con $\mathrm{SiC}$. En ella se puede observar claramente una huella de desgaste bastante importante. En la figura 4 se aprecia la acumulación de productos de desgaste ("wear debris") en el compuesto reforzado con $\mathrm{Al}_{2} \mathrm{O}_{3^{\prime}}$ así como la capa de deformación (tribolayer) formada, e incluso se observa un inicio de fisuración o delaminación de la capa amorfa de deformación en la parte izquierda. Por último, en la figura 5, se muestra un análisis de línea cualitativo (EDX) que nos muestra la presencia de $\mathrm{Cr}$ en la capa de deformación. Se puede apreciar un aumento importante de este elemento justo en correspondencia con esta capa en relación al resto de la línea de análisis. 




Figura 3. Sección transversal a la dirección de rodadura, mostrando huella de desgaste del material compuesto reforzado con $\mathrm{SiC}$.

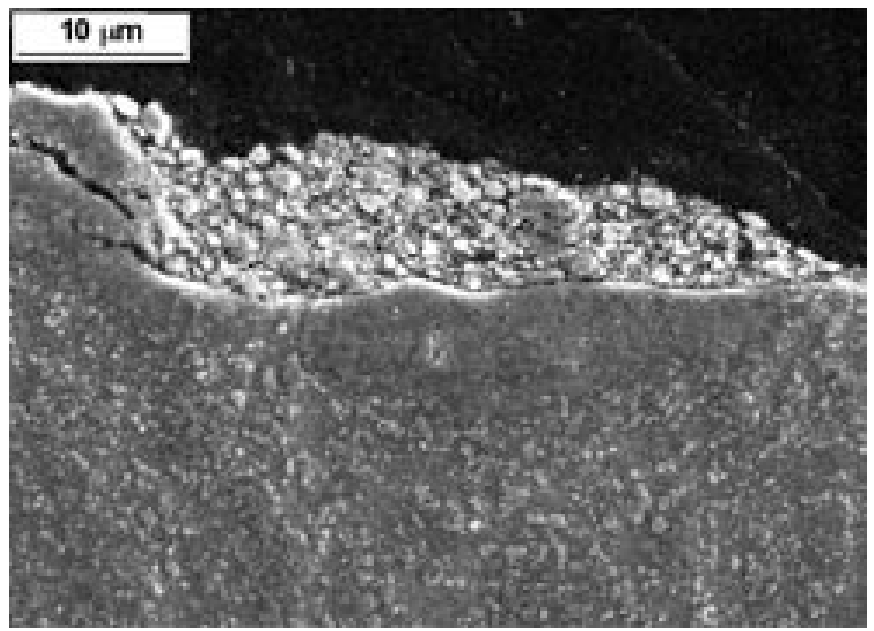

Figura 4. Sección transversal mostrando productos de desgaste y capa amorfa de deformación (tribolayer) del material compuesto reforzado con $\mathrm{Al}_{2} \mathrm{O}_{3}$.



Figura 5. Análisis EDX de línea a través de la zona amorfa de deformación (tribolayer) del material compuesto reforzado con $\mathrm{Al}_{2} \mathrm{O}_{3}$.

\section{DISCUSIÓN}

Los resultados obtenidos en el ensayo de tracción se acercan bastante a lo esperado por las características de los materiales compuestos. En nuestro caso, para fracciones volumétricas similares en todos los compuestos, el factor predominante que controla el endurecimiento es el tamaño de partícula. En efecto, en la literatura se observa que en general la resistencia de los compuestos reforzados con partículas depende con mayor intensidad de la fracción volumétrica del refuerzo que del tamaño de partícula (18-20). Sin embargo, cuando el tamaño de partícula es inferior a 5 micras, la presencia de las mismas pueden provocar un endurecimiento adicional por el temple del tratamiento térmico debido a los esfuerzos elásticos generados $(21,22)$. De este modo cabía esperar una mayor resistencia en los materiales compuestos reforzados con partículas más finas $\left(\mathrm{Si}_{3} \mathrm{~N}_{4^{\prime}} \mathrm{SiC}\right.$ y $\left.\mathrm{Al}_{2} \mathrm{O}_{3}\right)$. Esto ocurre así en los dos primeros casos pero no en el tercero, donde al contrario de lo esperado, se obtiene una resistencia inferior incluso a la alcanzada por la matriz sin reforzar. La explicación de este hecho habría que buscarla en los fenómenos de precipitación de la matriz. En efecto, la cinética de precipitación en el proceso de envejecimiento de la matriz se ve acelerada en general por la mayor velocidad de crecimiento de los precipitados, que en el caso de las aleaciones de la familia Al-Mg-Si, viene controlada por velocidad de difusión de los átomos de $\mathrm{Mg}$ (23-25). Esta velocidad aumenta debido a la presencia de un mayor número de dislocaciones producidas por el pequeño tamaños de los refuerzos en le caso del refuerzo de $\mathrm{Al}_{2} \mathrm{O}_{3}$ (26). De esta manera se produce un endurecimiento más rápido, lo que para el tratamiento efectuado (no optimizado para el compuesto) se traduce en un sobreenvejecimiento y por lo tanto en una caída de propiedades mecánicas.

Con respecto al comportamiento frente a desgaste de los distintos materiales estudiados, diferentes estudios han llegado a la conclusión ya apuntada en la introducción que en los materiales compuestos los dos factores que más influencian la resistencia al desgaste son el porcentaje de refuerzo y el tamaño del mismo $(1,9-12)$. Como en los compuestos estudiados la fracción volúmica de refuerzo empleada se mantiene constante e igual al 15\%, las diferencias observadas deben ser debidas, fundamentalmente al tamaño de los refuerzos. En efecto, los resultados de desgaste de la figura 1 demuestran que el éste es menor cuanto mayor es el tamaño de las partículas de refuerzo. Los dos compuestos con tasas de desgaste muy bajas, los reforzados con $\mathrm{B}_{4} \mathrm{C}$ y $\mathrm{Si}_{3} \mathrm{~N}_{4^{\prime}}$ tienen efectivamente un tamaño medio de partícula $(17 \mu \mathrm{m}$ y $6.6 \mu \mathrm{m}$ respectivamente) que es muy superior al de los compuestos con reforzados $\mathrm{Al}_{2} \mathrm{O}_{3}$ y $\mathrm{SiC}$ (en ambos casos con un diámetro inferior a las $2 \mu \mathrm{m}$ ). Así mismo vemos como la naturaleza de las partículas cerámicas no parece afectar en gran medida a los resultados, obteniéndose por ejemplo tasas de desgaste muy similares para éste último caso, donde tanto el tamaño como la morfología de partícula son bastante semejantes.

Con respecto al coeficiente de rozamiento, resulta mucho más complicado establecer una correlación entre volumen, tamaño y/o forma de refuerzo. Incluso en la literatura se encuentran casos en los el coeficiente de rozamiento para los compuestos reforzados con partículas cerámicas o bien es mayor que el de la matriz sin reforzar (9) o bien es menor (5) o bien, dependiendo de la sistemática del ensayo, una veces es mayor y otras menor para el mismo material (27). En nuestro estudio en el caso de los materiales reforzados con partículas tradicionales $\left(\mathrm{Al}_{2} \mathrm{O}_{3}\right.$ y $\left.\mathrm{SiC}\right)$ el coeficiente de rozamiento encontrado es superior al de la matriz sin reforzar, mientras que para los otros refuerzos cerámicos estudiados éste es inferior. Cabe destacar el bajo valor de coeficiente de rozamiento encontrado para el material compuesto reforzado con partículas de $\mathrm{Si}_{3} \mathrm{~N}_{4^{\prime}}$ que podría estar relacionado con la morfología de las partículas en forma de placas o escamas (tipo flake). 
Con respecto a los mecanismos de desgaste, después del análisis microscópico realizado, se han encontrado indicios que tanto el desgaste abrasivo como cohesivo están presentes en las muestras estudiadas, para las condiciones del ensayo. Así mismo, una importante transferencia del material de contacto $(\mathrm{Cr})$ ha tenido lugar en todos los casos, incorporándose a la capa amorfa de deformación. Este resultado concuerda con otras referencias, como en $(16,24)$, donde en ensayos de desgaste de materiales compuestos reforzados con partículas cerámicas tenía lugar una transferencia del material del disco (counterpart), en estos casos $\mathrm{Fe}$, a la capa de deformación (transfer layer o tribolayer). El mecanismo de formación de esta capa de deformación con altos contenidos en $\mathrm{Cr}$ puede ser resumido como sigue. En las primeras etapas de desgaste, se producen desprendimientos de partículas duras de refuerzo, que pasan a actuar como abrasivo (three-body wear) entre la muestra y el disco. Debido a la alta dureza de estos refuerzos, se produce el desgaste abrasivo del disco (así como de la muestra), incorporándose tanto material del disco como nuevo procedente de la muestra a los productos de desgaste. Por último, y debido a las altas presiones de contacto entre la muestra y disco, se embeben en la misma formando una capa con una elevada deformación y con contenidos apreciables de $\mathrm{Cr}$ procedente del disco.

\section{CONCLUSIONES}

El desgaste de los materiales compuestos de matriz de aluminio reforzados con partículas cerámicas del tipo $\mathrm{B}_{4} \mathrm{C}, \mathrm{TiB}_{2^{\prime}}, \mathrm{Si}_{3} \mathrm{~N}_{4^{\prime}} \mathrm{Al}_{2} \mathrm{O}_{3} \mathrm{y}$ $\mathrm{SiC}$ es menor que con respecto a la matriz sin reforzar.

La tasa de desgaste viene influenciada, a paridad de porcentaje de refuerzo, por el tamaño de las partículas del mismo, obteniéndose tasas menores para tamaños de partículas mayores, siendo independiente de su naturaleza.

Se han obtenido dispares variaciones del coeficiente de rozamiento con respecto a la matriz sin reforzar: en el caso de los compuestos reforzados con $\mathrm{Al}_{2} \mathrm{O}_{3}$ y $\mathrm{SiC}$ éste aumenta mientras que en el caso de aquellos reforzados con $\mathrm{TiB}_{2}, \mathrm{~B}_{4} \mathrm{C}$ y $\mathrm{Si}_{3} \mathrm{~N}_{4}$ ha resultado inferior.

Los mecanismos de desgaste producen la formación de una capa de deformación con un elevado contenido en $\mathrm{Cr}$ procedente de la erosión superficial por las partículas cerámicas del disco de contacto utilizado en el ensayo.

\section{BIBLIOGRAFÍA}

1. M. Roy, B. Venkataraman, V.V. Bhanuprasad, Y.R. Mahajan, G. Sundararajan, "The effects of particulate reinforcement on the sliding wear of aluminium matrix composites", Metallurgical Transactions 23A, pp. 2833-2847, (1991).

2. P.K. Rohatgi, Y. Liu, S. Ray, "Friction and wear of metal-matrix composites", ASM Handbook, Friction, Lubrication and Wear Technology, Vol. 18, ASM, pp. 801-811, (1992).

3 A. Martín, M.A. Martínez, J. Llorca, “Wear of SiC-reinforced Al-matrix composites in the temperature range $20-200^{\circ} \mathrm{C}^{\prime}$, Wear, Vol. 193, pp. 169-179, (1995).

4 M.K. Surappa, P.K. Rohatgi, "Preparation and properties of cast aluminiumceramic particle composites", Journal of Materials Science, Vol. 16, págs. 983-993, (1981).

5 F.M. Hosking, F. Folgar-Portillo, F.Wunderlin, R. Mehrabian, “Composites of aluminium alloys: fabrication and wear behaviour", Journal of Materials Science, Vol. 17, págs. 477-498, (1982).
6 K.J. Bhansali, R. Mehrabian, "Abrasive wear of aluminium matrix composites", Journal of Metals, Vol. 32, págs. 30-34, (1982).

7 Y.M. Pan, M.E. fine, H.S. Cheng, "Wear mechanisms of aluminium-based metal matrix composites under rolling and sliding contact", Tribology of Composite Materials, P.K. Rohatgi, B.G. Blau, C.S. Yust, Eds. ASM, págs. 93-101, (1990).

8 I.M. Hutchings, "Abrasive and erosive wear of metal matrix composites", Proceedings of the 2nd European Conference on Advanced Materials, Euromat '91, Cambridge, UK, T.W. Clyne and P.J. Whiters Eds., Institute of Metals, págs. 56-64, 1991.

9 A. Sato, R. Mehrabian, "Aluminium matrix composites: fabrication and properties", Metallurgical Transactions, Vol. 7B, págs. 443-451, (1976).

10 K. Anand, Kishore, "On the wear of aluminium-corundum composites", Wear, Vol. 85, págs. 163-169, (1983).

11 A.T. Alpas, J. Zhang, "Effect of microstructure (particulate size and volume fraction) and counterface material on the sliding wear resistance of particulate-reinforced aluminium matrix composites", Metallurgical and Materials Transactions, Vol. 25A, págs. 969-983, (19949.

12 S.V. Nair, J.K. Tien, R.C. Bates, "SiC -reinforced aluminium matrix composites", International Materials Reviews, Vol. 20, págs. 275-290, (1986).

13 T.W. Clyne, P.J. Withers, "An Introduction to Metal Matrix Composites". Cambridge University Press. Cambridge, (1993).

14 M.D. Salvador, N. Martínez, C. Ferrer, J. Oñoro y J.M. Ruiz, “Development of Al-Mg-Si alloys reinforced with Boron Carbide Particles", Euro PM2001, Proceedings of the PM 2001, Vol. 4, pp. 76-81, Ed. European Powder Association, Niza, 2001.

15 N. Martínez, V. Amigó, M.D. Salvador, C. Ferrer, “Caracterización microestructural y mecánica de la aleación AA6061 reforzada con partículas de diboruro de titanio por vía pulvimetalúrgica", Matcomp01, pp. 762-772, Ed. A. Fdez. Canteli, F.J. Belunze, F. Paris, Gijón, (2001).

16 N. Martínez, V. Amigó, M.D. Salvador, D. Busquets y C. Ferrer, “Wear behavior of ceramic particles reinforced aluminum matrix composites", Congress on Powder Metallurgy and Particulate Materials, PM2TEC2002, CD-Rom, Orlando, junio 2002.

17 C.E. da Costa, W. Zapata, J.M. Torralba, J.M. Ruiz Prieto, V. Amigó, “P/M MMCs base aluminium reinforced with $\mathrm{Ni}_{3} \mathrm{Al}$ intermetallic made by mechanical alloying route", Mater. Sci. Forums, 217-222, pp. 1859-1864, (1996).

18 "Aluminum-Matrix Composites", pp160-179, en "Aluminum and aluminum alloys", Ed. J.R. Davis, ASM International, Ohio, 1993.

19 D.J. Lloyd, "Particle Reinforced Aluminium and Magnesium Matrix Composites", International Materials Reviews, Vol. 39, núm. 1, págs. 1-23, (1994).

20 A. Rabiei, B. N. Kim, M. Enoki, T. Kishi, "Fracture behavior in $6061 \mathrm{Al}$ alloy matrix composites with different reinforcements" Materials Transaction JIM, vol. 37, No. 5, pp. 1148- 1155, (1996).

21 P.J. Withers, W.M. Stobbs, O.B. Pedersen, "The Application of the Eshelby Method of Internal Stress Determination to Short Fibre Metal Matrix Composites", Acta metall., Vol.37, No.11, pp.3061-3084, (1989).

22 M. Taya, K.E. Lulay, D.J. Lloyd. Strengthening of a Particulate Metal Matrix Composite by Quenching, Acta metall.mater. 39, pp. 73-87, 1991. Transaction JIM, vol. 37, No. 5, pp. 1148- 1155, (1996).

23 P. Merle, D. Dafir, S.M. Seyed Reyhani, "Study of the influence of the reinforcement on precipitation kinetics in a 6061/SiCp composite", Proceedings of the 9th International Conference on Composite Materials (ICCM/9), A. Miravete Ed., vol. I, pp. 401-408, Madrid, España, julio 1993.

24 I. Dutta, S.M. Allen, J.L Hafley, "Effect of reinforcement on the aging response of cast $6061 \mathrm{Al}-\mathrm{Al} 2 \mathrm{O} 3$ particulate composites", Metallurgical Transactions A, vol. 22, núm. 11, pp. 2553-2563, (1991).

25 G.A. Edwards, J.Y.Yao, M.J Couper, G.L. Dunlop, “The influence of ceramic particles on precipitation and age hardening in an Al2O3/Al-6061 metal matrix composite", The 3rd International Conference on Aluminium Alloys (ICAA3), L. Arnberg, O. Lohne, E. Nes, N. Ryum, Eds., vol. 1, pp. 525-530, Trondheim, Noruega, Junio 1992.

26 T. Das, P.R. Munroe, S. Bandyopadhyay, "The effect of $\mathrm{Al}_{2} \mathrm{O}_{3}$ particulates on the precipitation behaviour of 6061 aluminium-matrix composites", Journal of Materials Science, vol. 31, pp. 5351-5361, (1996).

27 B. Venkataraman, G Sundarajan, "The sliding wear behaviour of Al-SiC particulate composites - I. Macrobehaviour", Acta Mater. Vol. 44, No. 2, pp. 451-460, (1996).

Recibido: 1.2 .03

Aceptado: 30.11 .03 